\title{
Avaliação da mutagenicidade de Piper methysticum L. f. no sistema methG1 em Aspergillus nidulans
}

PALIOTO, G.F. ${ }^{1 *}$; ROCHA, C.L.M.S.C. ${ }^{2}$

1Universidade Tecnológica Federal do Paraná, Rua Marcilio Dias, 635, CEP: 86812-460, Apucarana-Brasil *gfppalioto@yahoo.com.br 2Universidade Estadual de Maringá, Avenida Colombo 5.790, CEP: 87020-900, Maringá-Brasil

\begin{abstract}
RESUMO: O extrato seco da raiz de Piper methysticum L. f. Forster (PIPERACEAE), a kava-kava, é usado no tratamento de diversos problemas envolvendo ansiedade como um dos sintomas. Por não causar dependência, sedação e ter ação ansiolítica, muitas pessoas têm recorrido a kava-kava para auxiliá-las no emagrecimento. Isto pode levar ao consumo indiscriminado da planta e acarretar riscos, pois todo medicamento fitoterápico deve respeitar limites de doses. Um risco na utilização de plantas medicinais é a toxicidade e, dentro deste, a mutagenicidade. Como a mutagenicidade está relacionada com a carcinogenicidade tornase importante testar este potencial na kava-kava. Assim, o objetivo deste trabalho foi avaliar o potencial mutagênico do extrato seco da raiz de $P$. methysticum no sistema meth 1 em Aspergillus nidulans. A linhagem utilizada foi a biA1meth G1, auxotrófica para biotina e metionina. Conídios dormentes de colônias crescidas por cinco dias foram tratados com soluções da kavakava nas concentrações de $0,35 \mathrm{mg} \mathrm{mL}^{-1}$ e $3,5 \mathrm{mg} \mathrm{mL}^{-1}$, e depois de $24 \mathrm{~h}$, semeados em meio seletivo contendo metionina, para análise dos sobreviventes, e sem metionina, para a análise dos mutantes. Os números de sobreviventes e mutantes dos tratamentos foram comparados aos do controle. Os resultados indicaram que o extrato da raiz da kava-kava é mutagênica, pois a freqüência de mutação dos tratamentos foi maior que da mutação espontânea, porém não ocorrendo diferença significativa entre as doses.
\end{abstract}

Palavras-chave: Piper methysticum L. f., kava-kava, mutagenicidade, sistema methG1, Aspergillus nidulans

ABSTRACT: Mutagenic evaluation of Piper methysticum L. f. in Aspergillus nidulans methG1 system. The dry root extract of Piper methysticum L. f. Forster (Piperaceae), kavakava, is used as to treat several health problems involving anxiety symptoms. As it causes no addiction, it can be applied as a sedative and anxiolytic. Many people have been relying on kava-kava as an auxiliary treatment. This can lead to an indiscriminate plant consumption and lead to risks, because all phytotherapic medications must observe dosage limits. One risk in the folk medicinal plant use is toxicity, and within it, mutagenicity. As mutagenicity is closely related to carcinogenicity, it is important to test the kava-kava mutagenicity potential. Thus, the purpose of this work was to test the mutagenicity of the dry root extract of $P$. methysticum in the meth 1 system of Aspergillus nidulans. The bia1meth 1 lineage, which is auxotrophic for biotine and methione, was used. Conidia from five-day-old colonies were collected and treated with kava-kava solutions at $0.35 \mathrm{mg} \mathrm{mL}^{-1}$ and $3.5 \mathrm{mg} \mathrm{mL}^{-1}$ concentrations and, after $24 \mathrm{~h}$, they were planted in selective growth medium with and without methione, in order to analyze the survivors and mutants, respectively. The number of survivors and mutants analyzed under effect of the treatments was compared with the control. The results indicated that the kava-kava dry root extract is mutagenic, since the mutation frequency of the treatments was higher than spontaneous mutation, however, there were no differences between the doses tested.

Keywords: Piper methysticum L. f., kava-kava, mutagenicity, methG1 system, Aspergillus nidulans

Recebido para publicação em 05/11/2010

Aceito para publicação em 12/11/2012

Rev. Bras. PI. Med., Campinas, v.15, n.3, p.347-351, 2013. 


\section{INTRODUÇÃO}

A espécie Piper methysticum L. f. (Forster) da família das Piperáceas, conhecida como kavakava, é uma planta originária das Ilhas Sandwich, Hawaii. Possui raiz cinzenta por fora e branca por dentro, com gosto picante, aromático, adstringente e contendo uma substância aquosa (Cruz, 1979; Lebot et al., 1992).

A kava-kava, antes de tudo, era utilizada em rituais nas ilhas de origem pela extração de suco narcótico das raízes. Hoje os extratos da raiz são usados no tratamento de diversos fins, pois os princípios ativos se combinam entre si apresentando, assim, efeito sinérgico (Williamson, 2001; Clouatre, 2004). Produtos contendo kavaína sintético não podem ser vistos como análogos aos do extrato vegetal, já que a biodisponibilidade é altamente influenciada pelos demais componentes do extrato (Digermann, 1995).

O grupo ativo atua no sistema nervoso central (Carlini, 2003), sendo a kava-kava utilizada principalmente no tratamento da ansiedade (Billia et al. 2002; Rex et al. 2002; Ernst, 2004; Sorrentino et al., 2006), depressão de grau leve (Cagnacci et al., 2003) e insônia (Volz e Kieser, 1997). As kavalactonas, os principais constituintes da planta, apresentam ação significativa como relaxante muscular-esquelética. No entanto, os mecanismos de ação das kavalactonas no sistema nervoso central ainda não são claros, existindo na literatura relatos contraditórios sobre a interação das lactonas com o receptor GABA (Simões et al., 1999).

Ensaios clínicos controlados com resultados positivos para ansiedade indicam a administração de setenta a cem miligramas de extrato enriquecido, administrado três vezes ao dia, pelo período de quatro semanas (Lehman et al., 1996). Porém estudos mais amplos, envolvendo maior número de pacientes são considerados necessários para estabelecer a efetividade nas indicações propostas, como medicamento sedativo e ansiolítico (Abell, 1997).

Recentemente, o extrato da kava-kava foi comercialmente proibido pela agência Food and Drug Administration dos Estados Unidos, por ter sido relatado casos de distúrbios hepáticos e renais em função de seu consumo (Bilia et al, 2002; Clayton, 2007; Denham et al, 2002). Muitos dos casos de falência hepática foram associados aos produtos contendo extratos de kava com álcool ou acetona (Currie e Clough, 2003). Contudo, Sorrentino et al. (2006) não encontraram nenhum sinal de toxicidade de extratos etanólicos em tratamentos com ratos Wistar.

Por não causar dependência nem sedação e também atuar contra a ansiedade, muitas pessoas têm recorrido a kava-kava para auxiliá- las no emagrecimento, já que na maioria dos casos a ansiedade leva a fome. $E$ isto pode levar ao consumo indiscriminado da planta, e acarretar riscos, pois qualquer medicamento fitoterápico ou sintético deve respeitar limites de doses.

Um dos riscos na utilização de plantas medicinais é a toxicidade e, dentro deste tópico, a mutagenicidade. Como a mutagenicidade está relacionada com o potencial carcinogênico é relevante testar este potencial do extrato seco da raiz de $P$. methysticum, que foi o objetivo do presente trabalho.

\section{MATERIAL E MÉTODO}

\section{Linhagem}

A linhagem utilizada para o sistema methG1 foi a biA1methG1, originada de Glasgow (Escócia). Essa linhagem é deficiente para biotina (cromossomo I) e metionina (cromossomo IV). Ela possui duas vantagens, não tem translocações e a mutação biA1 confere-lhe maior permeabilidade da parede celular.

\section{Meios de cultura}

Os meios de cultura foram preparados segundo Pontercorvo et al. (1959), Clutterbuck (1974) e Rocha (1997). Para o cultivo da linhagem para posterior tratamento foi utilizado o meio completo (MC). O meio seletivo (SM) para análise dos mutantes foi o meio mínimo (MM) suplementado com biotina $\left(0,02 \mu \mathrm{g} \mathrm{mL}^{-1}\right)$. O meio para teste de sobrevivência (SMM) foi preparado com a mesma composição do SM adicionando metionina (50 $\mu \mathrm{g}$ $\mathrm{mL}^{-1}$ ).

\section{Preparo da solução da kava-kava}

Os extratos secos da raiz de kava-kava foram obtidos em uma farmácia de manipulação, na forma de cápsulas, como sendo para um consumidor. As cápsulas foram abertas assepticamente e armazenadas em frascos estéreis. Foram preparadas duas concentrações com extrato seco da raiz da kava-kava em água destilada esterilizada: $0,35 \mathrm{mg} \mathrm{mL}^{-1}$ (T1) e $3,5 \mathrm{mg} \mathrm{mL}^{-1}$ (T2). As concentrações foram definidas de acordo com a maior quantidade solúvel de extrato (T2).

Como controle positivo $(\mathrm{C}+)$, para a validação do teste, foi realizado a exposição por 1 segundo a luz ultravioleta, como descrito em Palioto et al. (2010).

\section{Tratamento}

Conídios de colônias crescidas por cinco dias em $\mathrm{MC}$ a $37^{\circ} \mathrm{C}$ foram coletados em solução 
$0,01 \%$ Tween 80 , acrescido de $0,09 \% \mathrm{NaCl}$, agitados e filtrados em lã de vidro. Após a estimativa da quantidade de esporos em câmara hematimétrica, a suspensão foi dividida em três tubos. Dois tubos para o tratamento com as soluções da kava-kava e outro para o controle, onde foi adicionado água destilada esterilizada. Após 24 horas de tratamento, os esporos foram lavados por 3 vezes com salina por centrifugação a 4.000 rpm, por 10 minutos.

Foi realizado o plaqueamento em meio seletivo. Para estimativa de sobrevivência foram feitas diluições adequadas e inoculado $0,1 \mathrm{~mL}$ da suspensão em dez placas de SMM para cada tubo (tratamentos e controle) e incubados por três dias a $37^{\circ} \mathrm{C}$. Para análise dos mutantes foram inoculados $0,1 \mathrm{~mL}$ da suspensão sem diluição em dez placas de SM para cada tubo (tratamentos e controle) e incubados por cinco dias a $37^{\circ} \mathrm{C}$. Todo procedimento foi repetido três vezes independentemente.

\section{Análise estatística}

A análise estatística foi feita segundo Munson \& Goodhead (1977), adaptado para o sistema methG1 por Scott et al. (1982). Cada concentração do extrato seco foi analisado em separado, utilizando-se a média do número de conídios viáveis e da média da freqüência de mutação das três repetições.

O critério para a conclusão de positivo/ negativo é feita pela correlação linear entre a freqüência de mutação e o logaritmo neperiano da sobrevivência, de acordo com a equação:

$M_{C}-M_{T}=-m x \ln \left(S_{C} / S_{T}\right)$,

onde: $M_{C}$ - frequência de mutação no

controle; $M_{T}$ - frequência de mutação do tratamento;
$S_{C}$ - sobrevivência no controle; e $S_{T}$ sobrevivência no tratamento. Desta equação resulta o valor de $\mathrm{m}$ da respectiva concentração em análise. Para efeito de comparação, são calculados dois valores de $\mathrm{m}\left(\mathrm{m}_{\mathrm{C}}{ }_{\mathrm{C}}\right.$ e $\mathrm{m}_{\mathrm{C}}{ }_{\mathrm{c}}$ ), em que os valores de $\left[\mathrm{M}_{\mathrm{C}}-\mathrm{M}_{\mathrm{T}}\right]$ correspondem ao valor da frequência de mutação do controle em análise multiplicado por 2 e por 4 , respectivamente, e o valor de $\ln \left(S_{C} / S_{T}\right)$, é arbitrariamente fixado em 4. Se $m_{T}>m^{\prime}{ }_{C}$, a substância é considerada mutagênica. Se $m_{C}^{\prime}<m_{T}<m_{C}$, o resultado não é estatisticamente significativo.

\section{RESULTADO E DISCUSSÃO}

Observando a Tabela 1 para os tratamentos $0,35 \mathrm{mg} \mathrm{mL}^{-1}$ e $3,5 \mathrm{mg} \mathrm{mL}^{-1}$ das soluções de kava-kava, nota-se que a freqüência de mutação, representada pela razão entre o número de mutantes por mililitro e o número de conídios viáveis por mililitro é significativamente maior nos tratamentos quando comparada com a frequência de mutação do controle em cada experimento. O controle positivo da luz ultravioleta confirma a sensibilidade do teste para a detecção de mutações.

$\mathrm{Na}$ análise estatística (Tabela 2) são mostradas que as diferenças são significativas, pois os valores de $m_{T_{1}}$ e $m_{T 2}$ são maiores que o valor de $\mathrm{m}_{\mathrm{c}}$. Da mesma forma, a diferença dos dois valores de $\mathrm{m}$ dos tratamentos não é significativa, não caracterizando efeito de dose.

Para a determinação da mutagenicidade da kava-kava foi utilizado o sistema meth $G 1$ no fungo filamentoso $A$. nidulans. Este teste tem sido muito utilizado para avaliação do potencial mutagênico de inúmeros agentes físicos e químicos (Scott et al.,

TABELA 1. Resultados obtidos nos tratamentos e controle de conídios dormentes da linhagem biA1methG1, com $0,35 \mathrm{mg} \mathrm{mL}^{-1}$ (T1) e 3,5 $\mathrm{mg} \mathrm{mL}^{-1}$ (T2) da solução do extrato seco de raiz de Piper methysticum.

\begin{tabular}{|c|c|c|c|c|}
\hline Experimento & Grupos & $\begin{array}{c}\text { Número de conídios } \\
\text { viáveis } \times 10^{5} / \mathrm{mL}\end{array}$ & $\begin{array}{c}\text { Número de } \\
\text { mutantes } / \mathrm{mL}\end{array}$ & $\begin{array}{l}\text { Número de mutantes/ } \\
10^{6} \text { conídios viáveis }\end{array}$ \\
\hline \multirow[t]{3}{*}{1} & Controle & 110,2 & 22,0 & 0,2 \\
\hline & $\mathrm{T} 1$ & 105,7 & 43,0 & 4,0 \\
\hline & $\mathrm{T} 2$ & 72,3 & 68,0 & 9,4 \\
\hline \multirow[t]{3}{*}{2} & Controle & 183,0 & 78,0 & 4,3 \\
\hline & $\mathrm{T} 1$ & 131,7 & 95,0 & 7,2 \\
\hline & $\mathrm{T} 2$ & 121,9 & 84,0 & 7,0 \\
\hline \multirow[t]{3}{*}{3} & Controle & 240,0 & 136,0 & 6,0 \\
\hline & $\mathrm{T} 1$ & 190,0 & 163,0 & 8,6 \\
\hline & T2 & 72,8 & 130,0 & 17,9 \\
\hline \multirow[t]{3}{*}{ Média } & Controle & 177,7 & - & 3,5 \\
\hline & $\mathrm{T} 1$ & 142,5 & - & 6,6 \\
\hline & $\mathrm{T} 2$ & 89,0 & - & 11,4 \\
\hline
\end{tabular}

Rev. Bras. Pl. Med., Campinas, v.15, n.3, p.347-351, 2013. 
TABELA 2. Análise estatística dos resultados mostrando que os valores de $\mathrm{m}$ do controle (que representa a mutação espontânea) é menor que os valores de m de T1 e T2 (que representam a mutação em presença da solução do extrato seco da raiz de Piper methysticum nas concentrações de $0,35 \mathrm{mg} \mathrm{mL}^{-1}$ e $3,5 \mathrm{mg} \mathrm{mL}^{-1}$, respectivamente).

\begin{tabular}{ccc}
\hline \multicolumn{3}{c}{ Tratamento* $^{*}$} \\
\hline $\mathbf{C}+$ & $\mathrm{T} 1$ & $\mathrm{~T} 2$ \\
\hline $\mathrm{m}^{\prime}{ }_{\mathrm{C}}=3,40$ & $\mathrm{~m}_{\mathrm{C}}=1,75$ & $\mathrm{~m}_{\mathrm{C}}=1,75$ \\
$\mathrm{~m}_{\mathrm{C}}=6,8$ & $\mathrm{~m}_{\mathrm{C}}=3,50$ & $\mathrm{~m}_{\mathrm{C}}=3,50$ \\
$\mathrm{~m}_{\mathrm{T}}=26,70^{* *}$ & $\mathrm{~m}_{\mathrm{T}}=14,09^{* *}$ & $\mathrm{~m}_{\mathrm{T}}=14,35^{* *}$
\end{tabular}

${ }^{*} \mathrm{C}+$ (controle positivo) 1 segundo de exposição dos conídios à luz ultravioleta (PALIOTO, et al. 2010); T1 - 0,35 $\mathrm{mg} \mathrm{mL}^{-1}$; T2 $3,5 \mathrm{mg} \mathrm{mL}^{-1} ;{ }^{* *} \mathrm{o}$ tratamento é considerado mutagênico se o $\mathrm{m}_{\mathrm{T}}$ for maior que o $\mathrm{m}_{\mathrm{c}}$.

1982; Rocha \& Azevedo, 1986; Zonetti \& Rocha, 2000; Baroni-Rodrigues, 2001; Rodrigues et al., 2003; Rezende et al., 2004; Palioto et al., 2010).

Os resultados encontrados demonstraram claramente o potencial mutagênico da raiz de $P$. methysticum, preparado nas concentrações 0,35 $\mathrm{mg} \mathrm{mL}^{-1}$ e $3,5 \mathrm{mg} \mathrm{mL}^{-1}$, por meio do tratamento de conídios dormentes, no sistema meth 1 em $A$. nidulans. Este resultado é muito importante devido ao fato da maioria dos trabalhos com a kava-kava se limitarem a ação ansiolítica ou (hepato)tóxica.

As kavalactonas da raiz da kava-kava foram identificadas e são consideradas os constituintes responsáveis pelos efeitos farmacológicos da planta (Bilia et al., 2002; Singh e Singh, 2002). Jhoo et al. (2007) testaram a mutagenicidade de 6 kavalactonas isoladas da raiz de kava-kava no umu teste, em Salmonella typhimurium TA1535/ pSK 1002. Nenhuma das kavalactonas apresentou mutagenicidade neste teste, que detecta mutações de ponto. O sistema methG1 também detecta mutações de ponto e, os resultados positivos encontrados, apontam o sinergismo entre os compostos da raiz.

Com relação a citotoxicidade, Jhoo et al. (2006) encontraram nas frações aquosas da raiz de 79,6 a $102 \%$ de viabilidade celular, sendo o composto responsável pela citotoxicidade identificado, a flavokavaina B. Esses resultados estão de acordo com os resultados encontrados neste trabalho. Em todas as repetições, T1 e T2 apresentaram menor quantidade de conídios viáveis em comparação com o controle. Na media, T1 apresentou $80,19 \%$ de conídios viáveis e T2 50,08\%, mostrando um efeito de dose para a citotoxicidade.

Com relação a carcinogenicidade, Former et al. (2006) demonstraram que derivados de $P$. methysticum inibiram a ativação do fator de necrose tumoral á, uma molécula que desempenha importante função na resposta inflamatória e carcinogênica. A consequência da expressão gênica desregulada deste fator é caracterizada por doenças infecciosas, inflamatórias e, inclusive, o desenvolvimento de uma neoplasia (Anderson et al., 2004).

Antes de 1998, os extratos de kava-kava eram considerados seguros como uma droga ansiolítica alternativa. Contudo, em janeiro de 2003 os extratos da kava-kava foram banidos em alguns países devido ao registro de casos de insufiência hepática e renal (Bilia et al., 2002; Denham et al., 2002; Clouatre, 2004). Estes casos de hepatotoxicidade podem ser causados pelas kavalactonas por meio de três mecanismos: inibição do citocromo P450, redução da glutationa no rim, ou inibição da atividade da enzima ciclooxigenase (Clouatre, 2004). Muitos dos casos de hepatotoxicidade são relacionados ao consumo de produtos contendo extratos de kava preparados por métodos de extração com solventes (Jhoo et al., 2006).

Assim, este trabalho mostrou a relevância do estudo das propriedades mutagênicas das plantas medicinais que, por serem consideradas "naturais" pela cultura popular, muitas vezes são utilizadas indiscriminadamente. Devido ao potencial mutagênico revelado neste trabalho, este deve ser testado em outros sistemas testes de avaliação de mutagenicidade para a devida comprovação.

\section{CONCLUSÃO}

De acordo com os dados obtidos neste estudo, podemos concluir que a solução do extrato seco da raiz de Piper methysticum possui potencial mutagênico no sistema methG1 em Aspergillus nidulans sem apresentar efeito de dose. Devido a mutagenicidade estar intimamente ligada a carcinogenicidade, o extrato seco da raiz da kavakava deve ser contra-indicado como medicamento fitoterápico, principalmente como droga alternativa para a ansiedade.

\section{REFERÊNCIA}

ABELL, S. Drug evaluation: kava. In: HUTCHISON, T.A.; SHAHAN D.R.; ANDERSON, M.L. (Eds.). DRUGDEX ${ }^{\circledR}$ System. Colorado: Thomson Micromedex, 1997. p.87-99.

ANDERSON, G.M. et al. Tumor necrosis factor-á in the pathogenesis and treatment of cancer. Current Opinion in Pharmacology, v.4, n.4, p.314-20, 2004.

BARONI-RODRIGUES, S. Avaliação do potencial antimutagênico dos cogumelos Agaricus blazei e Lentinula edodes no sistema metG1 em Aspergillus(=Emericella) nidulans. 2001. 20p. Monografia (Especialização em Genética Aplicada ao 
Ensino) - Universidade Estadual de Maringá, Maringá.

BILIA, A.R. et al. Kava-kava and anxiety: growing knowledge about the efficacy and safety. Life Sciences, v.70, n.22, p.2581-97, 2002.

CAGNACCI, A. et al. Kava-Kava administration reduces anxiety in perimenopausal women. Maturitas, v.44, n.2, p.103-9, 2003.

CARLINI, E.A. Plants and the central nervous system. Pharmacology Biochemistry and Behavior, v.75, n.3, p.501-12, 2003.

CLAYTON, N.P. et al. Immunohistochemical analysis of expressions of hepatic cytochrome P450 in F344 rats following oral treatment with kava extract. Experimental and Toxicologic Pathology, v.58, n.4, p.223-36, 2007.

CLOUATRE, D.L. Kava kava: examining new reports of toxicity. Toxicology Letters, v.150, n.1, p.85-96, 2004.

CLUTTERBUCK, A.J. Aspergilluns nidulans. In: KING, R.C. (Ed). Handbook of Genetics. New York: Plenum Publishing. 1974. p.447-510.

CRUZ. G.L. Dicionário das plantas úteis do Brasil. Rio de Janeiro: Editora Civilização Brasileira, 1979. 412p.

DENHAM, A. et al. Kava - the unfolding story: report on a work-in-progress. Journal of Alternative and Complementary Medicine, v. 8, n.3, p. 237-63, 2002.

DIGERMANN, T. Phytopharmaka in alter: crataegus, ginkgo, hypericum und kava-kava. Pharmazeutische Zeitung, v.140, n.23, p.2017-24, 1995.

ERNST, E. Herbal remedies for anxiety - a systematic review of controlled clinical trials. Phytomedicine, v.13, n.3, p.205-8, 2006.

FOLMER, F. et al. Inhibition of TNF $\alpha$-induced activation of nuclear factor KB by kava (Piper methysticum) derivatives. Biochemical Pharmacology, v.71, n.8, p.1206-18, 2006.

JHOO, J.W. et al. In vitro cytotoxicity of nonpolar constituents from different parts of kava plant (Piper methysticum). Journal of Agricultural and Food Chemistry, v.54, p.3157"62, 2006.

JHOO, J.W. et al. Identification of c-glycoside flavonoids as potential mutagenic compounds in kava. Food Chemistry and Toxicology, v.72,n.2, p. 120-5, 2007.

LEBOT, V. et al. Kava-the Pacific drug. New Haven: Yale University Press, 1992. 159p.

LEHMAN, E. et al. Efficacy of a special kava extract (Piper methisticum) in patients with states of anxiety, tension and excitedness of non-mental oringin-A double-blind placebo-controlled study of four weeks treatmente. Phytomedicine, v.3, p.113-9, 1996.

MUNSON, R.J. et al. The relation between induced mutation frequency and cell survival - a theoretical approach and examination of experimental data for eukariotes. Mutation Research, v.42, p.145-60, 1977.

PALIOTO, N.F. et al. Efeito mutagênico de baixa dose de ultravioleta no sistema meth 1 em Aspergillus nidulans. Arquivo de Ciências da Saúde UNIPAR, v.14, n.1, p.49-54, 2010.

PONTERCORVO, G. et al. The genetics of Aspergilluns nidulans. Advances in Genetics, v.5, p.141-238, 1959.

REX, A. et al. Anxiolytic-like effects of Kava-Kava in the elevated plus maze test - a comparison with diazepam. Progress in Neuro-Psychopharmacology and Biological Psychiatry, v.26, n.5, p.855-60, 2002.

REZENDE, J.R. et al. Efeito antimutagênico do látex de Euphorbia no sistema metionina em Aspergillus nidulans. Acta Scientiarum, v.26, n.4, p.481-4, 2004.

ROCHA, C.L.M.S.C. Caracterização citológica, genética e molecular de um mutante para conidiogênese em Aspergilluns nidulans. 1997. 203p. Tese (Doutorado em Genética) - EASALQ/USP, Piracicaba.

ROCHA, C.L.M.S.C. et al. Use of methG1 reversion of Aspergilluns nidulans for the detection of mutagenicity of the pyrrolizidine alkaloid integerrimine. Revista Brasileira de Genética, v.9, p.393-405, 1986.

RODRIGUES, S.B. et al. Avaliação do potencial antimutagênico do cogumelo do sol (Agaricus blazei) no sistema methG1 em Aspergillus (=Emericella) nidulans. Acta Scientiarum, v.25, n.2, p.513-7, 2003.

SCOTT, B.R. et al. Aspergillus nidulans: Systems and results of tests for induction of mitotic segregation and mutation. II. Haploid assay systems and overall response of all systems. Mutation Research, v.98, p.49-94, 1982.

SIMÕES, C.M.O. et al. Farmacognosia: da planta ao medicamento. 3.ed. Porto Alegre/Florianópolis: Editora UFRGS/UFSC, 1999. 711p.

SINGH, Y.N.; SINGH, N.N. Therapeutic potential of kava in the treatment of anxiety disorders. CNS DRUGS, v. 16, n, 11, P.731-43, 2002.

SORRENTINO, L. et al. Safety of ethanolic kava extract: results of a study of chronic toxicity in rats. Phytomedicine, v.13, n.8, p.542-9, 2006.

VOLZ, H.P.; KIESER, M. Kava-kava extract ws 1490 versus placebo in anxiety disorders - a randomized placebo-controlled 25-week outpatient trial. Pharmacopsych, v. 30, n. 1, p. 1-5, 1997

WILLIAMSON, E.M. Synergy and other interactions in phytomedicines. Phytomedicine, v.8, n.5, p.401-9, 2001.

ZONETTI, P.C. et al. Mutagenicity of benlate in methG1 system in Aspergillus (=Emericella) nidulans. Cytologia, v.65, p.305-12, 2000. 